\title{
Appropriateness of Parametric Bootstrap System for Appraise Mean Time to Failure of a Problematical System
}

\author{
Amit Marmat ${ }^{1}$, Ritesh Nagar ${ }^{2 *}$ \\ ${ }^{I}$ Assistant Professor, Department of Electronics Engineering, School of Engineering \& Technology, Vikram \\ University, Ujjain, India \\ ${ }^{2}$ Research Scholar, Ujjain, India \\ *Corresponding author: rn4777@gmail.com
}

\begin{abstract}
This is a preview paper describes a study of an Appropriateness of Parametric Bootstrap system for appraise mean time to failure of a problematical system, where system having a different failure density functions. Mechanism for complete reliability and analysis with boot strapping can be done by Monte Carlo simulation technique for the complex network has been studied. The method is used with the bridge network. A bridge network is very useful while observation of faulty complex system and provides better accuracy. The result obtained has been compared with those obtained using and Monte Carlo simulation.
\end{abstract}

Keywords: Accuracy, Bootstrap, Failure density function, Mean time to failure (MTTF), Mean Time between Failures (MTBF), Monte Carlo simulation.

\section{Introduction}

The approach to establish different parameters of unwanted nature are taken in account and given in this paper; all these parameters are considered and taken as a group which causes failure of the system and average time of repair. Justification can be done by doing easy calculations of system. In this system the failed caused by transient in power system does not be taken in account. As we know that the different environmental condition effects on power systems is taken in this paper and due to this environmental effect on power system different types of errors, losses and even frailer of the system can be also possible even after using different protecting devices. Due to that the overall efficiency of the system is affected. In ideal conditions or normal conditions, the disused errors and losses are not taken in daily load variation curve. But, during practical or original conditions it can be seen that the buses are performing still at the loads side

To achieve this approach Monte Carlo simulation is one of the best technique because it has a tremendous ability to evaluate reliability ratios for average values even with all possible values. By using this technique gives the different details of the distribution system, like number of disturbance occurs are greater than specified value, number of utility doesn't bring back normal within defined time duration, etc.
These values are very important to calculate the disturbances at the utility side coast and average losses at the utility side. This values can also be used in different power generating system also to calculate their worthwhile production of electrical energy with economic boundaries of superficial in the electrical power systems. The worthwhile production of electrical power by wind energy is evaluated by simulating the functioning over a selected duration one-year period of time. To gain these types of lookalike structure or behavior a Monte Carlo simulation technique is best to use which generates the different data which is considered in wind power system and samples are taken in hourly basis and owing random behavior of both the load and wind. Therefore, this technique is implemented on different types of wind power station for their contribution by which we are able to find out their capacity and output of electrical energy generation for a system. The samples results given by this technique is taken in account which is ideal for estimating the reliability of the given systems. On comparing with other different types of techniques we found that the results are very irrelevant and system become complex. And different constant progressive for which bootstrap techniques have ability give prediction and variable collection, the techniques discussed is used extended the work with concluded in linear models and nonlinear models. In this system focus is mainly from the utilities viewpoint. By including the system reliability is also being an essential part having planning, designing, and control of systems. Many great innumerable models are used for reliability analysis. This is a Minimal Path sets (MP)-hard problem. The proposed Hybrid Monte Carlo method able to reach and gives the different reduction effect.

The main aim of using Hybrid Monte Carlo technique is that we are considering standard errors and more than $90 \%$ compact intervals of the system in which five basic statistics are used as a mechanism to get Bootstrap. By using this technique gives the better results which are very helpful to enhance the quality of the electric signals. In this mechanism we are able to predict the different stuff related by doing their assessment. While doing verity of methods can be used for finding out the values of 
different variables. In this research is done on two entities first is the overall operation of wind power and second is the critical industrial load. Some of the industrial loads are now a day's using their own power generating stations as a backup in the condition of power failure. The reliability issues associated with the stochastic nature of the new structured distribution systems. New reliability indices are proposed to judge the security and the adequacy of the service provided by the distributed generation. As there are the different methods to achieve the reliability by Monte Carlo simulation established by crossentropy method to estimate the generating capacity reliability of system. A comparative analysis of a conventional and cross phase unified power quality conditioning system and this system is able to handle power quality issues by compensating current and voltage simultaneously. Both the give techniques are very useful for the analysis of Power Quality for their enhancement, in this method all converters we use they are allied back to back to the dc side and shares a common dc-link capacitor. A risk-based system Alternators provides a preventive power system with wind power. This system provides a suitable method to reduce risk of loss in the Power System. Estimation is done by using sequential Markov Chain Monte Carlo (MCMC) simulation model. This technique also provides the information for the maintenance of power operators so that they should minimizes loss generated in power system so that the system will operate fluently and the condition of power failure can be avoided.

\section{Mean Time between Failure (MTBF) or Mean time to failures (MTTF)}

The Mean time between failures (MTBF) is the forecast of signal to proceed the time between native failure conditions occurs in electrical power system to reliable functioning of the system. MTBF will also calculate as the average time between failures of a system. The MTBF basically cast off for the repairable systems, but on other hand mean time to failure (MTTF) for a non-repairable system and gives the expected time to failure of the non-repairable system.

The MTBF for the complicated network, repairable systems, and failures are take that are not taken in account during designing and make system out of working condition and throw on the state for repair. So failures which are out of the box happens are repaired keeping it in unrepaired condition, and during this, system should be keeping online condition. But for this definition which includes different units taken down for routine scheduled maintenance in this duration the routine control of system is not considered.

\section{Reliability}

As we know that reliability of the system given the possibility of network to withstand and performing its function suitably under unusual conditions when it is subjected to risk.

The definition has four basic parts:
- Probability: It gives a numerical mathematical expression that lies on $[0,1]$. The term probability it is considered as a mathematical concept. By taking only the magnitude of the probability does not have lone has not any significance till some system description is taken in account.

- Time period: Reliability of a component i.e. probability of adequately performing in general is a function of time. Obviously reliability decreases as time period of study increases. It decreases from value 1 at $\mathrm{t}=0$ and 0 at $\mathrm{t}=\infty$ i.e. reliability function is a decreasing function of time.

- Adequate performance: The mathematical parameters has to be evaluated with respect to sufficient operation of the network. For this a professional engineer will take to decide these parameters of given network. It depends on the judgment of professional and want significant computational effort for a complex network. To achieve this power supply should be on desired voltage, current and frequency.

- Operating condition or operating environment: Reliability of a component will depend on the environment in which it is functioning. A personal computer put in a laboratory of the institute under air conditioner will have higher reliability than a hostel room without air-conditioner.

\section{Monte Carlo Simulation Technique}

Monte Carlo techniques are a wide group of algorithmic system which gives the recurrent odd samples to gives arithmetic results. The basic idea is to use chance to workout on the issues which cannot be unavoidable in this principle. This can be applied on actual and arithmetic problems but this technique is most useful with respect to other techniques where it is difficult to reach on different parameters of the samples. Monte Carlo methods are mostly used in three classes that are optimization, numerical integration, and generating draws from a cumulative distribution.

\section{Problematic System Analysis by Using Monte Carlo Simulation}

The power analysis by using Monte Carlo simulation, following method given as, in this method, we simulate a number of different native parameters which the effect the system under online condition, and gives numerous samples (at least 1000) that have approximate size and different structure like original data. The proportion of times the simulated effect is detected in the random samples is the statistical power to detect that effect from a given sample size. For observation, the numerous parameters were approximate based on the model of the actual data collected, except that the parameter for the effect of upper and lower limits at the equivalents in some of results which set to the size of the slowdown observed. Using normal distributions with these numerous parameters, near around 5000 data sets with the same system and system were simulated. Each set of data gives constant mixed effects of the model that is calculated using the same fixed and random parameters 
examine on the actual data. This gives the mean time to faultier of the signal while under different unwanted condition this can be achieved by bootstrapping of the signal, so that the different parameters help to maintain system stable and ability of the system to withstand.

\section{Conclusion}

Application of Monte Carlo simulation which gives a proper information how close the system can be observed by using MCS and also gives evaluating Mean Time to Failure (MTTF) and Reliability of a complex electrical network by using Monte Carlo simulation bootstrap technique. Each of the elements of the system are exponential distributed. This shows an improvement in Mean Time to Failure (MTTF) bootstrap technique and with the help of Monte Carlo simulation technique.

\section{References}

[1] Roy Billinton, R. N. Allan, "Introduction to Reliability Evaluation of Engineering System”, Springer International Edition, August 1992.

[2] R. Balguruswamy, "Reliability Engineering", Tata Mc Graw Hill 1984.

[3] D. P. Kothari, I. J. Nagrath, "Modern Power System Analysis", 3rd Edition Tata MC Graw Hill Ltd, 2006.

[4] G. J, Babu, "Bootstrapping statistics with linear combination of Chisquare as weak limit," The Indian journal of statistics, Vol .46, 1984, pp.85-93.

[5] G. Desrochers, M. Blanchard, "A Monte-Carlo simulation method for the economic assessment of the contribution of wind energy to power systems"', IEEE Power Engg. Review, 86WM 024-4 December 1986, pp. 50-56.

[6] B. Korver, "The Monte Carlo method and software reliability theory," 5th Simulation Conference, Singapore, February 18, 1994, pp.1-7.

[7] S. Bulteau, Khadiri, "A Monte Carlo simulation of the flow network reliability using importance and stratified sampling," Technical Report, March 1997, pp.1-17.

[8] J. P. C. Kleijnen. A. J. Feelders, "Bootstrapping and validation of Meta models in simulation", proce. of 1998 Winter Simulation Conference, May 1998, pp. 701-706.
[9] I. Pardoe, S. Weisberg, "An Introduction to Bootstrap methods using Arc," University of Minnesota, St. Paul, Technical Report Number 631, 2001, pp. 1-14.

[10] W. C. Yeh, "A new Monte Carlo method for the network reliability," ICITA 2002, pp.114-123.

[11] Skylar Lei, M. Smith, "Evaluation of several efron Bootstrap methods to estimate error measures for software metrics," Proce. of IEEE Canadian conference on electrical and computer engineering, 2002, pp .703-707.

[12] A. B. Huseby, M. Naustdal, I. D. Varli, "System reliability evaluation using Conditional Monte Carlo method," University of Oslo, Statistical Research Report, No. 2, January 2004.

[13] A. A. Chowdhury, D. O. Koval, "Reliability assessment of a backup gas turbine generation system for a critical industry load using a Monte Carlo simulation model," IAS 2004, pp. 2155-2161.

[14] S. Nikolovski, P. Maric, I. Mravak, "Reliability assessment of distribution networks using sequential Monte Carlo simulation," 18th International conference on Electricity distribution, Turin, 6-9 June 2005.

[15] Y. G. Hegazy, A. Mostafa, "Reliability Indices of Electrical distributed generation systems," Proce. of 5th WSEAS Int. Conference on Power Systems and Electromagnetic Compatibility, Greece, August 23-25, 2005, pp. 436-441.

[16] P. K. GOswami, S. Chowdhury, S. P. Chowdhury, Y. H. Song and J. K. Das, "Reliability evaluation of distribution system," UPEC 2007, pp. 158166

[17] S. Prasomphan, C. Lursinsap and S. Chiewchanwattana, "Imputing time series data by regional-gradient-guided bootstrapping algorithm," ISCIT, 2009, pp. 163-168.

[18] Ritesh Nagar, S. K. Bhatt, "A Comparative analysis of a Conventional and cross phase UPQC for enhancing the Power Quality," vol. 7, no. 7. IJSHRE, 2018.

[19] Yorlandys Salgado Durate, Janusz Szpytko et al., "Monte Carlo simulation model to coordinate the preventive maintenance scheduling of generating units in isolated distributed Power Systems," in Electric Power Systems Research, vol. 182, May 2020.

[20] A. M. Leite da Silva, R. A. G. Fernandez, C. Singh, "Generating capacity Reliability evaluation based on Monte Carlo simulation and cross-entropy method," IEEE Transactions on Power System, vol. 25, no. 1, February 2010, pp. 129-137.

[21] Jin Chu Wu, Alvin F. Martin \& Raghu N. Kacker, "Monte Carlo studies of bootstrap variability in ROC analysis with data dependency," Communications in Statistics - Simulation and Computation, vol. 48, no. 2, pp. 317-333, 2019. 